\title{
No Serological Evidence of Trachoma or Yaws Among Residents of Registered Camps and Makeshift Settlements in Cox's Bazar, Bangladesh
}

\author{
Gretchen M. Cooley, ${ }^{1}$ Leora R. Feldstein, ${ }^{2,3}$ Sarah D. Bennett, ${ }^{3}$ Concepcion F. Estivariz, ${ }^{3}$ Lauren Weil, ${ }^{4}$ Rajendra Bohara, ${ }^{5}$ \\ Maya Vandenent, ${ }_{6}^{6}$ ASM Mainul Hasan, ${ }^{6}$ Mohammad Saifuddin Akhtar, ${ }^{6}$ M. Salim Uzzaman, ${ }^{8}$ Mallick Masum Billah, ${ }^{8}$ \\ Laura Conklin, ${ }^{3}$ Daniel C. Ehlman, ${ }^{3}$ Kingsley Asiedu, ${ }^{7}$ Anthony W. Solomon, ${ }^{7}$ ASM Alamgir, ${ }^{8}$ Meerjady Sabrina Flora, ${ }^{8}$ and \\ Diana L. Martin ${ }^{1 *}$ \\ ${ }^{1}$ Division of Parasitic Diseases and Malaria, Centers for Disease Control and Prevention, Atlanta, Georgia; ${ }^{2}$ Epidemic Intelligence Service, Centers \\ for Disease Control and Prevention, Atlanta, Georgia; ${ }^{3}$ Global Immunization Division, Centers for Disease Control and Prevention, Atlanta, Georgia; \\ ${ }^{4}$ National Center for Immunization and Respiratory Diseases, Centers for Disease Control and Prevention, Atlanta, Georgia; ${ }^{5}$ World Health \\ Organization, Dhaka, Bangladesh; ${ }^{6}$ United Nations Children's Fund, Dhaka, Bangladesh; ${ }^{7}$ World Health Organization, Geneva, Switzerland; \\ ${ }^{8}$ Institute of Epidemiology, Disease Control and Research, Dhaka, Bangladesh
}

\begin{abstract}
Successful achievement of global targets for elimination of trachoma as a public health problem and eradication of yaws will require control efforts to reach marginalized populations, including refugees. Testing for serologic evidence of transmission of trachoma and yaws in residents of registered camps and a Makeshift Settlement in Cox's Bazar District, Bangladesh, was added to a serosurvey for vaccine-preventable diseases (VPDs) conducted April-May 2018. The survey was primarily designed to estimate remaining immunity gaps for VPDs, including diphtheria, measles, rubella, and polio. Blood specimens from 1- to 14-year-olds from selected households were collected and tested for antibody responses against antigens from Treponema pallidum and Chlamydia trachomatis using a multiplex bead assay to evaluate for serologic evidence of the neglected tropical diseases (NTDs) yaws and trachoma, respectively. The prevalence of antibodies against two C. trachomatis antigens in children ranged from $1.4 \%$ to $1.5 \%$ for Pgp 3 and $2.8 \%$ to $7.0 \%$ for CT694. The prevalence of antibody responses against both of two treponemal antigens (recombinant protein 17 and treponemal membrane protein A) tested was $0 \%$ to $0.15 \%$ in two camps. The data are suggestive of very low or no transmission of trachoma and yaws, currently or previously, in children resident in these communities. This study illustrates how integrated serologic testing can provide needed data to help NTD programs prioritize limited resources.
\end{abstract}

\section{INTRODUCTION}

Between late August 2017 and December 2017, more than 650,000 people moved into the Cox's Bazar area in Bangladesh, joining approximately 300,000 others who had arrived during earlier waves of migration. ${ }^{1}$ The two preexisting registered camps, Kutupalong and Nayapara, and Makeshift Settlements expanded with the new influx. Many partners are working to address the health issues facing this densely populated and vulnerable population. In the first 7 weeks of 2018 , large numbers of cases of acute watery diarrhea $(N=$ $36,533)$ and acute respiratory infection $(N=74,034)$ were reported. ${ }^{2}$ Measles and diphtheria outbreaks were also reported. ${ }^{3}$

From September 2017 to March 2018, vaccination campaigns were implemented to reduce the risk of transmission of measles, diphtheria, and other vaccine-preventable diseases (VPDs). ${ }^{4}$ To guide further vaccination activities, a household vaccination coverage and serosurvey was undertaken in April-May 2018 that included integrated serological surveillance using multiplex bead assays for targets linked to parasitic and neglected tropical diseases (NTDs) that have elimination goals. The assay panel included antigens specific for Chlamydia trachomatis and Treponema pallidum; ocular strains of $C$. trachomatis cause trachoma, and $T$. pallidum subspecies pertenue causes yaws.

Trachoma is targeted for elimination as a public health problem. ${ }^{5}$ Districts with $\geq 5 \%$ prevalence of the sign trachomatous inflammation-follicular (TF) in 1- to 9-year-olds require interventions that include antibiotic mass drug

*Address correspondence to Diana Martin, 1600 Clifton Road NE, Atlanta, GA 30329. E-mail: hzx3@cdc.gov administration to affected communities and efforts to address facial cleanliness and environmental improvement. ${ }^{6}$ Myanmar has eliminated trachoma (https://www.who.int/ southeastasia/news/detail/11-09-2020-myanmar-eliminatestrachoma-who), and Bangladesh is not thought to require interventions. ${ }^{7}$ However, displaced populations deserve special consideration. The ongoing high incidence of acute watery diarrhea in these communities suggests overcrowding and poor access to sanitation, conditions also found in trachomaendemic communities of other countries.

Myanmar was previously endemic for yaws, but like many countries that carried out truncated eradication campaigns in the mid-20th century, the current status of yaws in Myanmar is unknown. ${ }^{8}$ Bangladesh is not known to have ever been endemic for yaws. ${ }^{8}$ After the discovery that a single oral dose of azithromycin could effectively treat yaws, ${ }^{9}$ the WHO revived the goal of yaws eradication, aiming for complete interruption of transmission-the absence of new cases-globally by 2020.

Serologic testing for antibody responses against $C$. trachomatis antigens is gaining traction as a potential approach for conducting surveillance in areas that have achieved elimination criteria for trachoma and ceased interventions. Seroprevalence of antiC. trachomatis antibodies typically increases with age among 1to 9-year-olds in areas with ongoing transmission ${ }^{10-12}$ but remain relatively flat, with low seroconversion rates, ${ }^{13}$ in the absence of transmission ${ }^{14-16}$ and in settings where the presence of TF does not correlate with ocular $C$. trachomatis infection. ${ }^{17,18}$ Serologic testing is standard for yaws diagnosis: the $T$. pallidum particle agglutination (TPPA) assay reflects a history of $T$. pallidum infection, whereas nontreponemal tests such as rapid plasmin reagin (RPR) detect antibodies against host molecules released in response to infection, giving an indication of current or recent 
exposure. These tests together are diagnostic for active yaws or syphilis (caused by T. pallidum sp. pallidum; serologically indistinct from $T$. pallidum pertenue). We have recently adapted recombinant antigens from $T$. pallidum for use on the multiplex bead assay platform, with good correlation between responses to the antigen recombinant protein17 (rp17) and TPPA tests, and between responses to treponemal membrane protein $A(T m p A)$ and RPR titers. ${ }^{19}$

In this study, we measured antibody responses to $C$. trachomatis and T. pallidum antigens to opportunistically evaluate serologic evidence of yaws and trachoma transmission, respectively, in the population in two settlements of Cox's Bazar, Bangladesh. We used samples collected in an integrated serosurvey that had been primarily designed to estimate remaining immunity gaps for VPDs.

\section{METHODS}

Sampling. A full description of the methods and sampling used during the integrated household coverage and serologic survey in April-May 2018 is published elsewhere. ${ }^{4}$ Representative samples of households in Nayapara Registered Camp, Kutupalong Registered Camp, and the Makeshift Settlements were selected. In Nayapara and Kutupalong, simple random sampling of households was conducted using household registration lists. In the Makeshift Settlements, a multistage cluster-sampling approach was undertaken.

In each selected household, one child aged 6 months to 6 years and one child aged 7 to 14 years were randomly selected for interview. Selected children aged 1 to 14 years were eligible for dried blood spot (DBS) specimen collection. Sample size estimates assumed 1) $65 \%$ of households would have at least one child aged 6 months to 6 years $(60 \%$ for DBS specimen collection, given that age was further restricted to those aged 1-6 years) and $60 \%$ to $65 \%$ would have at least one child aged 7 to 14 years; 2) a household nonresponse rate (i.e., no one home) of $20 \%$ in the Registered Camps and $5 \%$ in the Makeshift Settlements; 3) a child nonresponse rate of $1 \%$ for all children for the survey component of the assessment; and 4) a $10 \%$ refusal rate for DBS specimen collection among children aged 1 to 6 years and $20 \%$ for children aged 7 to 14 years. Target enrollment was estimated assuming vaccination coverage with three doses of diphtheria-containing vaccine, and the proportion of children with protective antibody levels for tetanus was $50 \%$.

An interview with an available caregiver was conducted in Rohingya in or near the home, using a standardized questionnaire that included questions about demographics, length of time in Registered Camps or Makeshift Settlements, vaccination history, and recent health concerns. The expected enrollment was 1,089 children aged 6 months to 6 years and 1,055 children aged 7 to 14 years. Because data collection in Nayapara took place during Ramadan and many children were expected to observe fasting, DBS samples were not collected from 7- to 14-year-olds there.

Enrollment in Kutupalong was stopped early because the camp leadership did not want the community to participate in the survey at that time.

From eligible children, we collected three to four drops of blood using a single finger prick per child (expected number of children to be enrolled for DBS sample collection: 914 aged
1-6 years and 787 aged $7-14$ years). Blood was collected onto Whatman 903 Protein Saver Cards (GE Healthcare, Pittsburgh, PA). One large drop of blood was applied to each circle $(\approx 70 \mu \mathrm{L} / \approx 13 \mathrm{~mm}$ diameter), with up to four circles filled per child. The DBS were air-dried for at least 4 hours with protection from light, dust, and insects in plastic boxes inside dark back packs. After drying, DBS were placed in sealable plastic bags with a 5-g desiccant sachet and humidity indicator card, then stored in larger sealable plastic bags with additional desiccant and a humidity indicator card, which were checked daily and changed weekly. DBS were sent to CDC laboratories in the United States for testing using a multiplex bead assay to measure antibody levels against a panel of antigens from several VPDs and parasitic and neglected tropical diseases, including the antigens Pgp3 and CT694 from C. trachomatis and rp17 and TmpA from T. pallidum.

Multiplex bead Aassay. A 3-mm circular punch, assumed to have a $50 \%$ hematocrit, was taken from a blood-filled circle of each card and eluted in $125 \mu$ L Elution Buffer (1X phosphate buffered saline [PBS], 0.05\% sodium azide, 0.3\% Tween-20; Calbiochem, Burlington, MA) overnight at $4{ }^{\circ} \mathrm{C}$ in a $96-$ well plate. Elutions were diluted to a final serum concentration of 1 : 400 in in Buffer B (1X PBS, 0.5\% polyvinyl alcohol, 0.8\% polyvinylpyrrolidone, $0.5 \%$ casein, all Sigma, Burlington MA; $0.3 \%$ Tween-20, $0.02 \%$ sodium azide, filtered with a $0.2 \mu \mathrm{m}$ filter) containing $3 \mu \mathrm{g} / \mathrm{mL}$ of Escherichia coli extract and incubated overnight at $4^{\circ} \mathrm{C}$.

Specimens were incubated in duplicate in a 96-well plate with beads, followed by incubation with anti-human immunoglobulin (Ig)G and IgG4 (both Southern Biotech, Birmingham $A L)$ and streptavidin-linked R-phycoerythrin reporter (Invitrogen, Waltham, MA) [20]. Antibody binding was reported as median fluorescence intensity (MFI) using a MAGPIX instrument (Luminex, Austin, TX). Background from a DBS blank was subtracted from the (MFI-BG). Specimens having a coefficient of variation of $>15 \%$ between the MFI-BG of duplicate wells for any bead region were repeated.

Cutoffs for seropositivity were determined by receiver operator characteristic curves. For $C$. trachomatis antigens, the positive panel consisted of sera from 101 children from the United Republic of Tanzania previously classified as antibodypositive; the negative panel was derived from 74 U.S.-born 1to 9-year-olds residing in New York who tested negative for Chlamydia spp. and antichlamydial antibodies using the microimmunofluorescence assay. ${ }^{20}$ The rp17 positive panel consisted of 39 samples from 4- to 15-year-olds from yawsendemic areas who were positive by TPPA, and the negative panel consisted of 20 samples from individuals living in yawsendemic areas who had tested negative by both TPPA and RPR, plus 74 samples from U.S.-born and -resident 1- to 6year-olds assumed to be nonexposed by virtue of living in nonendemic country $(N=119)$. Because anti-TmpA antibodies may decrease after resolution of infection, ${ }^{19,21}$ the positive panel was restricted to samples that tested positive for TPPA and had a RPR titer of $\geq 4(N=15)$. The negative panel for TmpA consisted of 22 endemic-community samples that were TPPA-positive and RPR-negative, 47 that were both TPPA- and RPR-negative, and 74 samples from children from nonendemic areas $(N=143)$.

Ethics. The survey received nonresearch determination from CDC. It was reviewed and approved by the Bangladesh Institute of Epidemiology, Disease Control and Research 
institutional review board. All participation was voluntary, and the objectives, participation time, and risks and benefits of participation were explained to each participant prior to collecting data. The caregiver of each child provided verbal consent for participation. Verbal assent was obtained from older children.

Data analysis. Data were analyzed using GraphPad Prism v7.02 (La Jolla, CA) and R studio (Boston, MA). For analysis of treponemal-specific antibody responses, data were stratified into 1- to 4-year-olds, which likely reflect current yaws transmission status, and 5- to 14-year-olds (or 5- to 6-year-olds for Nayapara), the age range most likely to be affected by yaws. Analysis of $C$. trachomatis-specific antibody responses was restricted to 1 - to 9-year-olds, the age range currently used for programmatic decision-making using clinical markers. Confidence intervals (Cls; 95\%) were determined for nonzero data points; when antibody prevalence was 0 , binomial exact calculation using one-sided $97.5 \%$ confidence intervals was used. Cls are not presented for data from Kutupalong, where sample collection was stopped early due to insecurity, because this truncated sampling introduces biases that prevent reliable estimation of uncertainty.

To estimate ocular C. trachomatis transmission intensity, we calculated the seroconversion rate (SCR) by fitting a simple reversible catalytic model to the measured seroprevalence, stratified into yearly age groups, using maximum likelihood methods. ${ }^{22}$ For these models, no seroreversion was assumed. SCRs were only estimated for $C$. trachomatis because seroprevalence to treponemal antigens is not anticipated to increase with age among 5- to 14-year-olds in endemic areas.

\section{RESULTS}

Figure 1 shows the location of each of the camps included in the survey. Table 1 summarizes enrollment for Nayapara, Kutupalong, and the Makeshift Settlements. Adequate DBS samples were collected from 657 children in the Makeshift Settlements and 273 children in Nayapara.

C. trachomatis-specific antibody responses. In Nayapara, the prevalence of antibody responses among 1- to 6year-olds $(N=273)$ was $1.5 \%$ (95\% Cl: $0.47-4.0)$ against Pgp3 and $7.0 \%$ (95\% Cl: 4.4-10.8) against CT694. In the Makeshift Settlements $(N=657)$, antibody responses among 1 - to 9year-olds was $1.4 \%(95 \% \mathrm{Cl}$ : $0.67-2.8)$ against Pgp3 and $2.8 \%$ (95\% Cl: 1.7-4.6) against CT694. The intensity of the antibody responses (in MFI-BG) by age is shown in Figure 3. The estimated SCR for Nayapara was 0.0043 per year $(95 \%$ Cl: 0.0032-0.0053) for Pgp3 and 0.029 per year $(95 \% \mathrm{Cl}$ : 0.026-0.031) for CT694. For the Makeshift Settlements, SCR was estimated to be 0.0033 per year (95\% Cl: 0.0028-0.0038) for Pgp3 and 0.011 per year (95\% Cl: 0.0096-0.013) for CT694 (Figure 4).

Treponemal-specific antibody responses. In Nayapara, prevalence of antibody responses among 1- to 6-year-olds $(N=273)$ was $2.4 \%(95 \% \mathrm{Cl}: 0-5.0)$ against rp 17, 0.37\% (95\% $\mathrm{Cl}: 0-2.3)$ against TmpA, and $0 \%(97.5 \% \mathrm{Cl}$ : 0-1.7\%) against both antigens (Table 2). In the Makeshift Settlements $(N=657)$,

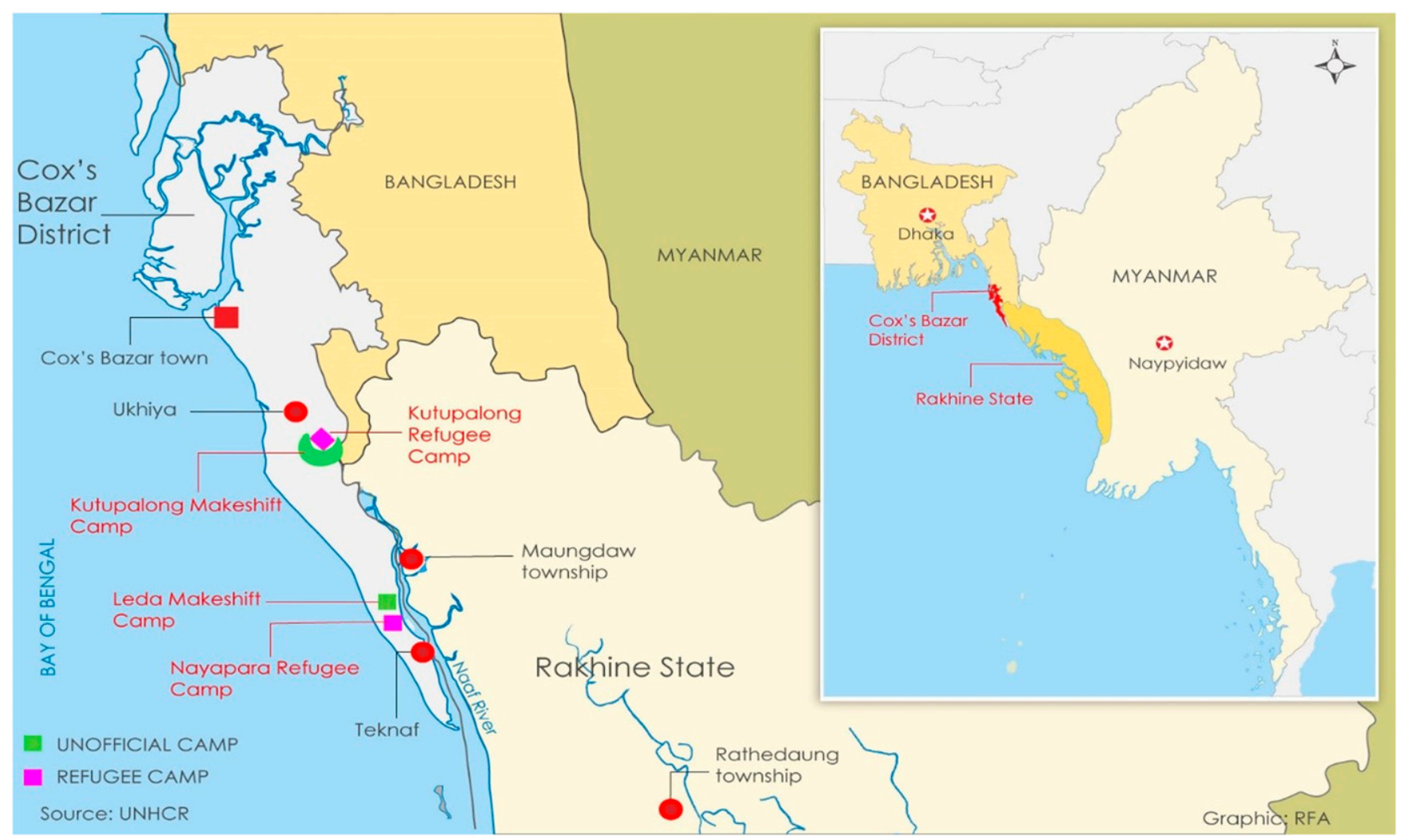

Figure 1. Location of sites in Cox's Bazar, Bangladesh. The boundaries and names shown and the designations used on this map do not imply the expression of any opinion whatsoever on the part of the World Health Organization concerning the legal status of any country, territory, city, or area or of its authorities, or concerning the delimitation of its frontiers or boundaries. This figure appears in color at www.ajtmh.org. 
TABLE 1

Enrollment and sex, by site

\begin{tabular}{|c|c|c|c|}
\hline & Makeshift Settlements & Nayapara & Kutupalong \\
\hline Number of households visited & 715 & 524 & 410 \\
\hline Number enrolled & 675 (94\%) & $483(92 \%)$ & 328 (80\%) \\
\hline Number not enrolled & $40(6 \%)$ & $41(8 \%)$ & $82(20 \%)$ \\
\hline Not at home & $39(5 \%)$ & $41(8 \%)$ & $20(5 \%)$ \\
\hline Refusal & $1(0.1 \%)$ & $0(0 \%)$ & $62(15 \%)$ \\
\hline Number of children enrolled & 829 & 638 & 394 \\
\hline Number eligible for DBS collection & 789 (95\%) & $281(44 \%)$ & $390(98.9 \%)$ \\
\hline Number with adequate DBS collected & $657(83 \%)$ & $273(97 \%)$ & $309(79 \%)$ \\
\hline Number without DBS collected & $132(17 \%)$ & $8(3 \%)$ & $81(21 \%)$ \\
\hline Not at home & $92(12 \%)$ & $6(2 \%)$ & $51(13 \%)$ \\
\hline Parent refused & $3(0.4 \%)$ & $0(0 \%)$ & $14(4 \%)$ \\
\hline Child refused & $15(2 \%)$ & $1(0.4 \%)$ & $14(4 \%)$ \\
\hline Insufficient blood draw & $20(3 \%)$ & $1(0.4 \%)$ & $0(0 \%)$ \\
\hline Other reason (e.g., child visibly sick) & $2(0.3 \%)$ & $0(0 \%)$ & $2(1 \%)$ \\
\hline Sex (female) & $317(44 \%)$ & $126(46 \%)$ & $142(46 \%)$ \\
\hline
\end{tabular}

DBS $=$ dried blood spots. This table was adapted from Feldstein et al. ${ }^{4}$

prevalence of antibody responses among 1- to 14-year-olds $(N=675)$ was 3.3\% (95\% Cl: $2.1-5.0)$ against rp17, $0.91 \%$ (95\% Cl: 0.3-2.0) against TmpA, and 0.15\% (95\% Cl: 0-0.8\%) against both antigens. Table 2 shows the antibody data by site and stratified into 1- to 4-year-olds and 5- to 14-year-olds, the age group typically included in active yaws case identification. The intensity of the antibody responses (in MFI-BG) by age is shown in Figure 2.

Data from abbreviated survey in Kutupalong. Of 410 households visited before discontinuation of the survey in Kutupalong, $15 \%$ refused to participate and $5 \%$ were not at home. In the remaining 328 households, adequate DBS samples were collected from 309 children. No children were seropositive for antibodies to $\mathrm{rp} 17$ and only one child was seropositive for antibodies to TmpA. Three children were seropositive for antibodies to Pgp3 and 11 children were seropositive for antibodies to CT694. Table 1 shows enrollment information and demographic information from the households visited before cessation of the survey in Kutupalong. Supplemental Tables 1 and 2 show raw antibody data for treponemal and C. trachomatis antigens, respectively.

\section{DISCUSSION}

Multiplex bead assays allow testing for antibodies against multiple antigens from a variety of pathogens in the same well, saving considerable laboratory processing and testing time and facilitating integrated surveillance efforts. This could be especially useful for surveillance of NTDs, which, as their "neglected" designation indicates, have significantly fewer resources devoted to their control than their importance as causes of human suffering would demand: NTDs are among the most common infections worldwide, affecting an estimated 2.9 billion people. ${ }^{23}$ In the current evaluation, testing for antibodies against the pathogens that cause trachoma and yaws was included as part of a larger serosurvey designed to measure vaccination coverage among children in communities in Cox's Bazar, Bangladesh. This survey was itself embedded in a SMART (Standardized Monitoring and Assessment of Relief and Transition) nutritional survey, which seeks to assess the nutritional status of the under-5 population and mortality rate of the entire population in situations of humanitarian crisis. $^{24}$ The data showed no serologic evidence for transmission of the pathogens causing yaws and trachoma in these communities.

The frequency of antibody responses to $C$. trachomatis antigens, especially Pgp3, in this population was exceptionally low, similar to that seen in U.S. children ${ }^{25}$ and children in districts previously endemic for trachoma in Nepal, ${ }^{16}$ the United Republic of Tanzania, ${ }^{15}$ and Ghana. ${ }^{26}$ In the three camps, the frequency of antibody responses was similar to or lower than the $6.2 \%(95 \% \mathrm{Cl}$ : $0.0-19.9 \%)$ threshold established in models used to predict trachoma as a public health problem. ${ }^{13}$ Where trachoma is a public health problem, the prevalence of anti-C. trachomatis antibodies typically increases with age in children. Models suggest SCRs $<0.015$ per year (95\% Cl: 0.0-0.49) correlate with TF < 5\% ${ }^{13}$; SCRs estimated for these camps was within that range or fell below that potential cutoff.

TABLE 2

Antibody responses to treponemal antigens $\mathrm{rp} 17$ and TmpA by site

\begin{tabular}{|c|c|c|c|c|c|c|c|c|}
\hline \multirow[b]{2}{*}{ Site } & \multirow[b]{2}{*}{ Age (years) } & \multirow[b]{2}{*}{ Number sampled } & \multicolumn{2}{|c|}{ Anti-rp17+ } & \multicolumn{2}{|c|}{ Anti-TmpA+ } & \multicolumn{2}{|c|}{$\begin{array}{c}\text { Anti-rp17 + and } \\
\text { anti-TmpA+ }\end{array}$} \\
\hline & & & $n$ & $\%(95 \% \mathrm{Cl})$ & $n$ & $\%(95 \% \mathrm{Cl})$ & $n$ & $\%(95 \% \mathrm{Cl})$ \\
\hline \multirow[t]{3}{*}{ Nayapara Registered Camp } & $1-4$ & 147 & 5 & $3.4(1.1-7.8)$ & 1 & $0.68(0-3.7)$ & 0 & $0(0-2.5)$ \\
\hline & $5-6$ & 126 & 3 & $2.4(0-5.0)$ & 1 & $0.37(0-2.3)$ & 0 & $0(0-17)$ \\
\hline & All & 273 & 8 & $2.9(0.9-4.9)$ & 2 & $0.73(0-1.7)$ & 0 & $0(0-1.1)$ \\
\hline \multirow[t]{3}{*}{ Makeshift Settlements } & $1-4$ & 246 & 13 & $5.3(2.8-8.9)$ & 2 & $0.81(0.1-2.9)$ & 1 & $0.4(0-2.2)$ \\
\hline & $5-14$ & 411 & 9 & $2.2(1.0-4.1)$ & 4 & $0.97(0.3-2.5)$ & 0 & $0(0-0.9)$ \\
\hline & All & 657 & 22 & $3.3(2.1-5.0)$ & 6 & $0.91(0.3-2.0)$ & 1 & $0.15(0-0.8)$ \\
\hline
\end{tabular}

$\mathrm{Cl}=$ confidence interval; $n=$ number positive; rp17 = recombinant protein17; TmpA = treponemal membrane protein A. When antibody prevalence was 0 , binomial exact calculation using onesided $97.5 \% \mathrm{Cl}$ was used. 

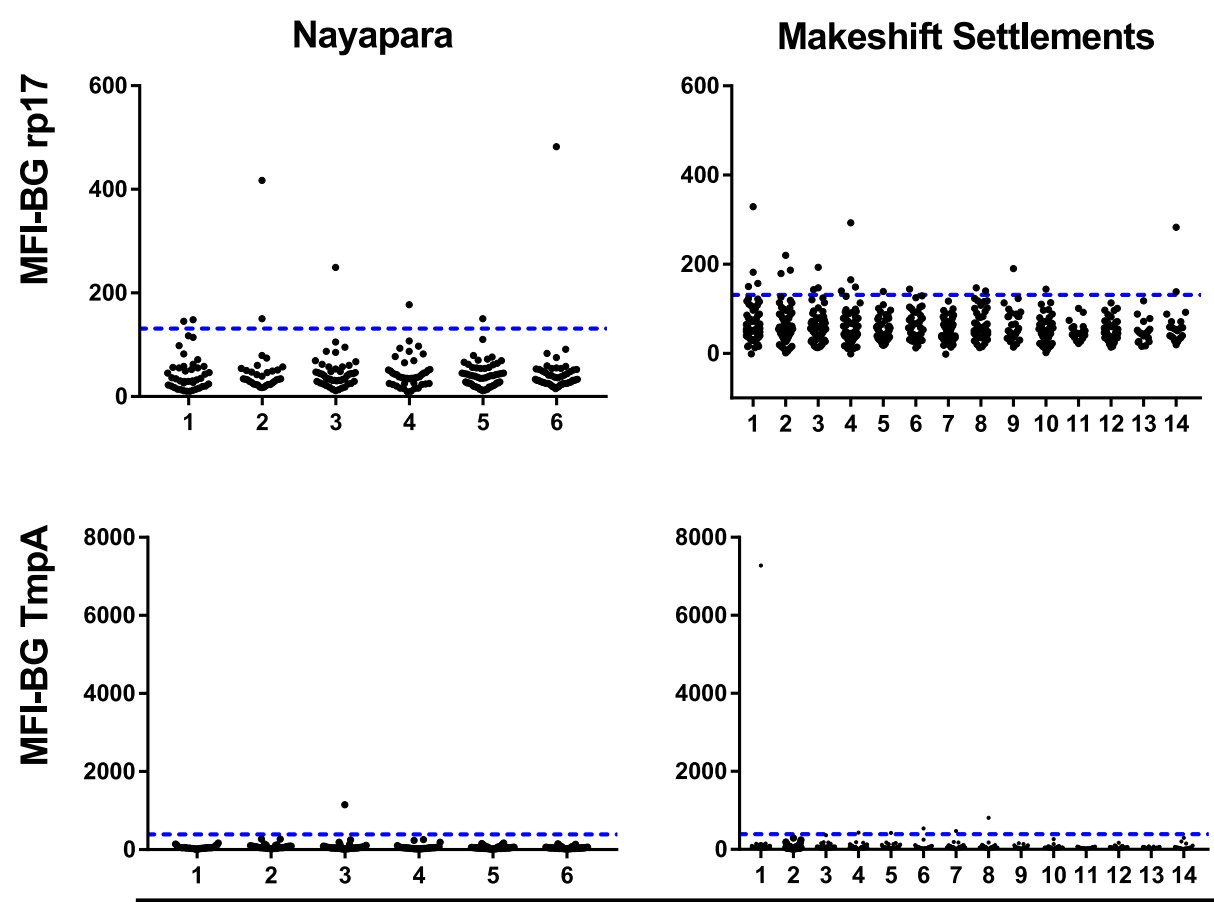

Age (years)

FIGURE 2. Intensity of antibody responses to the treponemal antigens rp17 (top) and TmpA (bottom) stratified by age in years up to 6 years for Nayapara Registered Camp (left) and up to 14 years for Makeshift Settlements (right). Horizontal lines indicate the cutoff for positivity. MFI-BG = median fluorescence intensity minus background. The upper range of the Y-axis scale differs for each antigen (600 for rp17 and 8,000 for TmpA, adjusted to be the same within each antigen to compare the two sites). TmpA = treponemal membrane protein A; $r p 17=$ recombinant protein17. This figure appears in color at www.ajtmh.org.

A potential limitation to interpreting these data is that in Nayapara, children aged 7 years and older were not included, potentially limiting our ability to detect possible increases in seroprevalence with age. Although having serological data on older children is ideal, community-based surveys in younger age groups $^{12}$ in which TF prevalence was $>5 \%$ have shown an increase in seroprevalence by age. Therefore, based on the available data, an increase in seroprevalence by age should still have been observable in 1- to 6-year-olds in Nayapara if ocular C. trachomatis transmission was ongoing. The prevalence of antibody responses against CT694 was consistently higher than those against Pgp3, although these responses tend to cluster around the cutoff, as seen in Figure 3. Although the interpretation of serological data for trachoma should be made with caution because these tests are not currently included in WHO guidance for trachoma surveillance, ${ }^{27}$ the combination of very low seroprevalence to $C$. trachomatis antigens and low SCR, especially against Pgp3, is strongly suggestive of low or no transmission of ocular $C$. trachomatis in these populations.

The WHO has set an eradication target for yaws by 2020 , but lack of data on where yaws is currently endemic and lack of funding for mapping necessitates creative approaches for determining where to prioritize surveys and interventions. ${ }^{28}$ One such approach is using integrated serological testing from samples collected from other surveys, as was done in this study. Use of recombinant antigens in an MBA is not part of the standard diagnostic criteria for yaws. However, TmpA has been shown to have high specificity for T. pallidum, and responses to TmpA decrease within a year of treatment ${ }^{21}$ and show good correlation with RPR titers. ${ }^{19} \mathrm{Rp} 17$ is an immunodominant antigen from $T$. pallidum ${ }^{29}$ with a reactive concordance of $94 \%$ to $99 \%$ against traditional treponemal tests. ${ }^{19}$ Only one of the children in the three sites under evaluation tested positive for antibody to both rp17 and TmpA antigens, suggesting little or no yaws in these populations.

The early cessation of data collection in Kutupalong limits the representativeness of the seroprevalence estimates for this camp. The number of antibody-positive individuals enrolled before data collection interruption, although not conclusive, are suggestive that children in Kutupalong have also been relatively infrequently exposed to the pathogens causing yaws and trachoma.

One challenge of integrated surveillance is ensuring that the sampling strategy for the targeted disease is epidemiologically appropriate for evaluating nontargeted diseases. In this survey, the numbers of households and 1- to 9-year-olds included overall were similar to those included in standard baseline trachoma surveys. ${ }^{30}$ Therefore, although the sampling is likely adequate for trachoma, it is likely suboptimal for yaws, so care should be taken not to overinterpret the lack of children with antitreponemal antibodies as a complete lack of yaws in this setting. Guidance on the sampling strategy that yaws programs should adopt in baseline surveys has not yet been generated; the eradication target, which requires demonstration of absence of infection transmission, makes this particularly challenging. However, within the limits of available guidance and subject to the caveats identified here, these data suggest that neither yaws nor trachoma is likely to be affecting this population. 

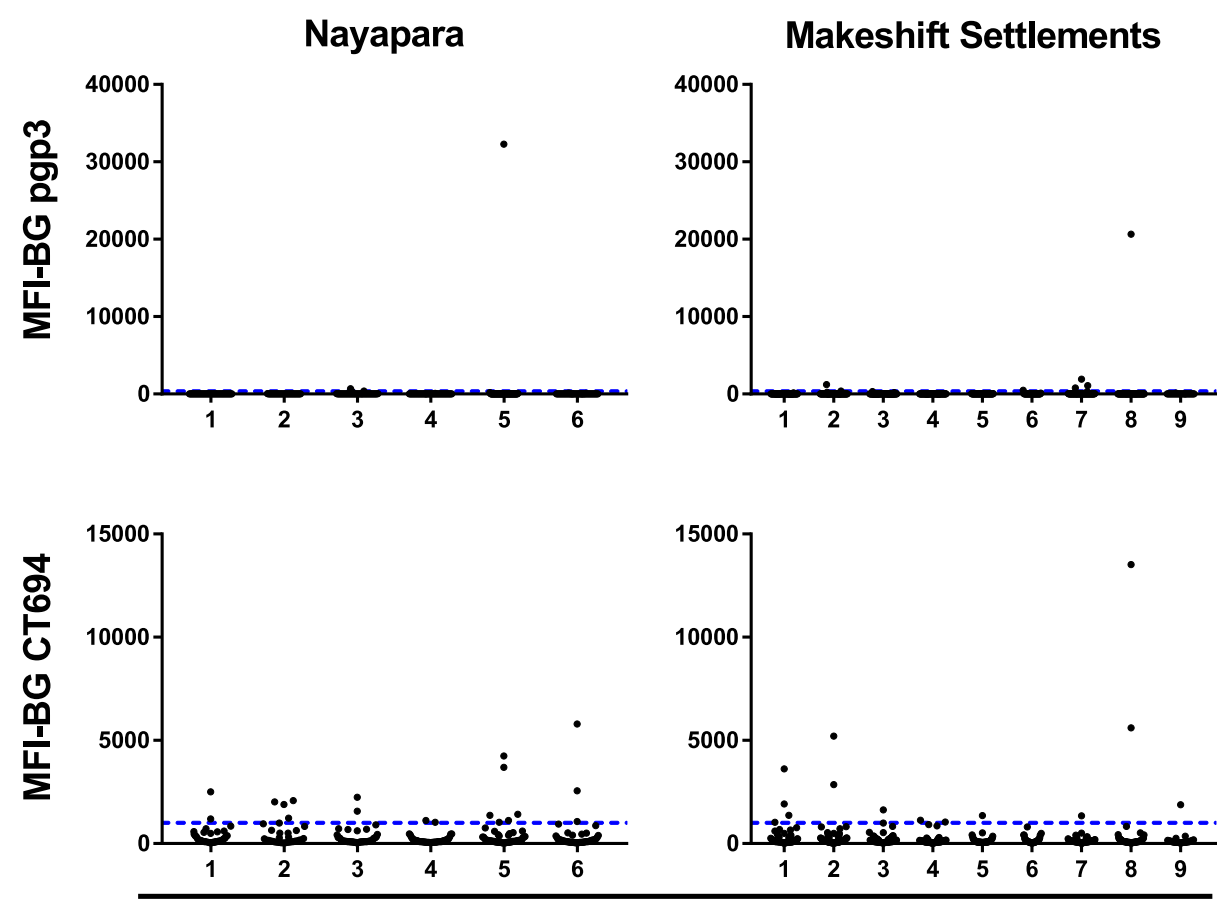

\section{Age (years)}

FIGURE 3. Intensity of antibody responses to the Chlamydia trachomatis antigens Pgp3 (top) and CT694 (bottom) stratified by age in years up to 6 years for Nayapara Registered Camp (left) and up to 9 years for Makeshift Settlements (right). Each dot represents an individual. Y-axis shows the intensity of the antibody response against each antigen measured in median fluorescence intensity (MFI) with background levels subtracted out (MFI-BG). The upper range of the Y-axis scale differs for each antigen (40,000 for Pgp3 and 15,000 for CT694, and then adjusted to be the same within each antigen to compare the two sites). The dashed blue line shows the seropositivity cutoff. CT =C. trachomatis; Pgp = plasmid gene product. This figure appears in color at www.ajtmh.org.

Received February 1, 2021. Accepted for publication March 16, 2021.

Published online May 3, 2021.

Notes: Supplemental tables appear at www.ajtmh.org. The World Health Organization holds the copyright to this article and has granted the publisher permission for the reproduction of this article.

Acknowledgments: We thank the field teams from the Bangladesh Institute of Epidemiology and Research, United Nations Children's Fund and Action Against Hunger, for their contributions to this survey. Funding for fieldwork was provided by the United Nations Children's Fund, and funding for the laboratory work was provided by the U.S. Centers for Disease Control and Prevention.

Disclaimer: The authors alone are responsible for the views expressed in this article and they do not necessarily represent the views, decisions or policies of the institutions with which they are affiliated.
Authors' addresses: Gretchen M. Cooley and Diana L. Martin, Division of Parasitic Diseases and Malaria, Centers for Disease Control and Prevention, Atlanta, GA, E-mails: xxd1@cdc.gov and hzx3@cdc.gov. Leora R. Feldstein, Epidemic Intelligence Service and Global Immunization Division, Centers for Disease Control and Prevention, Atlanta, GA, E-mail: nqw5@cdc.gov. Sarah D. Bennett, Concepcion F. Estivariz, Laura Conklin, and Daniel C. Ehlman, Global Immunization Division, Centers for Disease Control and Prevention, Atlanta, GA, E-mails: iyk3@cdc.gov, cge3@cdc.gov, dvj3@cdc.gov, and euh3@cdc.gov. Lauren Weil, National Center for Immunization and Respiratory Diseases, Centers for Disease Control and Prevention, Atlanta, GA, E-mail: nsi4@ cdc.gov. Rajendra Bohara, World Health Organization, Dhaka, Bangladesh, E-mail: boharar@who.int. Maya Vandenent, ASM Mainul Hasan, and Mohammad Saifuddin Akhtar, United Nations Children's Fund, Dhaka, Bangladesh, E-mails: mvandenent@unicef.org, ashasan@unicef.org, and msakhtar@unicef.org. M. Salim Uzzaman, Mallick Masum Billah, ASM Alamgir, and Meerjady Sabrina Flora, Institute of Epidemiology, Disease
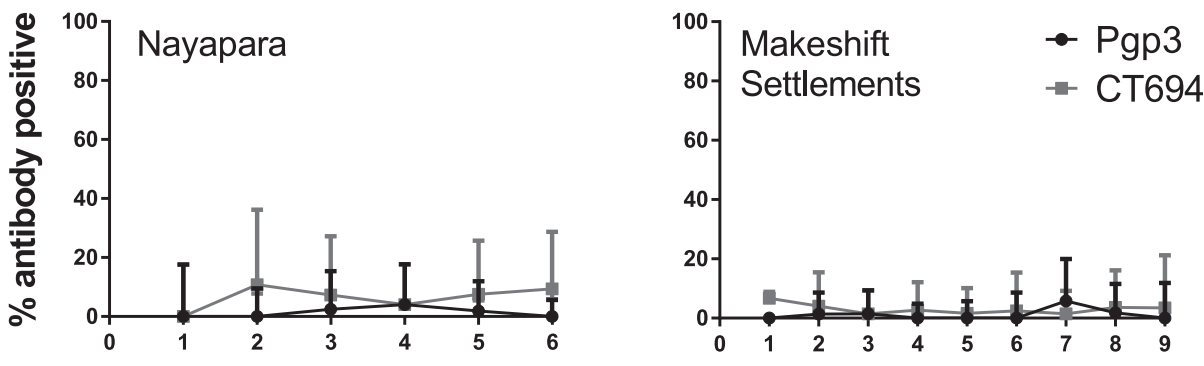

\section{Age (years)}

FIGURE 4. Seroprevalence of antibody responses to the Chlamydia trachomatis antigens Pgp3 (black line) and CT694 (gray line) by age. Vertical lines represent $95 \%$ confidence intervals. $C T=C$. trachomatis; Pgp = plasmid gene product. 
Control and Research, Dhaka, Bangladesh, E-mails msalimuzzaman@hotmail. com, drmasumbillah@yahoo.com, aalamgir@gmail.com, and meerflora@ yahoo.com. Kingsley Asiedu and Anthony W. Solomon, World Health Organization, Geneva, Switzerland, E-mails: asieduk@who.int and solomona@who.int.

This is an open-access article distributed under the terms of the Creative Commons Attribution (CC-BY) License, which permits unrestricted use, distribution, and reproduction in any medium, provided the original author and source are credited.

\section{REFERENCES}

1. Situation Report: Rohingya Refugee Crisis, InterSector Coordination Group. Cox's Bazar. Available at: https://reliefweb.int/report/ bangladesh/iscg-situation-report-rohingya-refugee-crisis-cox-sbazar-july-2020.

2. Summers A, Humphreys A, Leidman E, Van Mil LT, Wilkinson C, Narayan A, Mald, Miah ML, Cramer BG, Bilukha O, 2018. Notes from the field: diarrhea and acute respiratory infection, oral cholera vaccination coverage, and care-seeking behaviors of Rohingya Refugees-Cox's Bazar, Bangladesh, October-November 2017. MMWR Morb Mortal Wkly Rep 67: 533-535.

3. World Health Organization, 2017. Mortality and Morbidity Weekly Bulletin 8. Geneva, Switzerland: WHO.

4. Feldstein LR et al., 2020. Vaccination coverage survey and seroprevalence among forcibly displaced Rohingya children, Cox's Bazar, Bangladesh, 2018: a cross-sectional study. PLOS Med 17: e1003071.

5. World Health Organization, 1996. Future Approaches to Trachoma Control: Report of a Global Scientific Meeting, Geneva, Switzerland, June 17-20, 1996. Geneva, Switzerland: WHO.

6. Solomon AWZM, Kuper H, Buchan JC, Mabey DCW, Foster A, 2006. Trachoma Control: A Guide for Programme Managers. Geneva, Switzerland: World Health Organization.

7. World Health Organization. Global Health Observatory Data Repository. Geneva, Switzerland: WHO.

8. Mitja O et al., 2015. Global epidemiology of yaws: a systematic review. Lancet Glob Health 3: e324-e331.

9. Mitja O, Hays R, Ipai A, Penias M, Paru R, Fagaho D, de Lazzari E, Bassat $Q, 2012$. Single-dose azithromycin versus benzathine benzylpenicillin for treatment of yaws in children in Papua New Guinea: an open-label, non-inferiority, randomised trial. Lancet 379: 342-347.

10. Cama A et al., 2017. Prevalence of signs of trachoma, ocular Chlamydia trachomatis infection and antibodies to Pgp3 in residents of Kiritimati Island, Kiribati. PLoS Negl Trop Dis 11: e0005863.

11. Gwyn SE, Xiang L, Kandel RP, Dean D, Gambhir M, Martin DL, 2018. Prevalence of Chlamydia trachomatis-specific antibodies before and after mass drug administration for trachoma in community-wide surveys of four communities in Nepal. Am J Trop Med Hyg 98: 216-220.

12. Martin DL et al., 2015. Serological measures of trachoma transmission intensity. Sci Rep 5: 18532.

13. Pinsent $A$ et al., 2018. The utility of serology for elimination surveillance of trachoma. Nat Commun 9: 5444.

14. Migchelsen SJ et al., 2017. Serology reflects a decline in the prevalence of trachoma in two regions of The Gambia. Sci Rep 7: 15040.
15. West SK, Munoz B, Weaver J, Mrango Z, Dize L, Gaydos C, Quinn TC, Martin DL, 2016. Can we use antibodies to Chlamydia trachomatis as a surveillance tool for national trachoma control programs? Results from a district survey. PLoS Negl Trop Dis 10: e0004352.

16. West SK, Zambrano Al, Sharma S, Mishra SK, Munoz BE, Dize L, Crowley K, Gaydos CA, Rotondo LA, 2017. Surveillance surveys for reemergent trachoma in formerly endemic districts in Nepal from 2 to 10 years after mass drug administration cessation. JAMA Ophthalmol 135: 1141-1146.

17. Butcher $\mathrm{R}$ et al., 2018. Clinical signs of trachoma are prevalent among Solomon Islanders who have no persistent markers of prior infection with Chlamydia trachomatis. Wellcome Open Res 3: 14.

18. Cocks $\mathrm{N}$ et al., 2016. Community seroprevalence survey for yaws and trachoma in the Western Division of Fiji. Trans $R$ Soc Trop Med Hyg 110: 582-587.

19. Cooley GM et al., 2016. Evaluation of multiplex-based antibody testing for use in large-scale surveillance for yaws: a comparative study. J Clin Microbiol 54: 1321-1325.

20. Gwyn S, Cooley G, Goodhew B, Kohlhoff S, Banniettis N, Wiegand R, Martin DL, 2017. Comparison of platforms for testing antibody responses against the Chlamydia trachomatis antigen Pgp3. Am J Trop Med Hyg 97: 1662-1668.

21. Ijsselmuiden OE, Schouls LM, Stolz E, Aelbers GN, Agterberg CM, Top J, van Embden JD, 1989. Sensitivity and specificity of an enzyme-linked immunosorbent assay using the recombinant DNA-derived Treponema pallidum protein TmpA for serodiagnosis of syphilis and the potential use of TmpA for assessing the effect of antibiotic therapy. J Clin Microbiol 27: 152-157.

22. Drakeley $\mathrm{CJ}$ et al., 2005. Estimating medium- and long-term trends in malaria transmission by using serological markers of malaria exposure. Proc Natl Acad Sci USA 102: 5108-5113.

23. Hotez PJ, Molyneux DH, Fenwick A, Kumaresan J, Sachs SE, Sachs JD, Savioli L, 2007. Control of neglected tropical diseases. N Engl J Med 357: 1018-1027.

24. Action Against Hunger, 2018. Available at: https://actionagainsthunger. ca/program-areas/smart/. Accessed July 25, 2018.

25. Goodhew EB et al., 2012. CT694 and pgp3 as serological tools for monitoring trachoma programs. PLoS Negl Trop Dis 6: e1873.

26. Senyonjo LG et al., 2018. Serological and PCR-based markers of ocular Chlamydia trachomatis transmission in northern Ghana after elimination of trachoma as a public health problem. PLoS Negl Trop Dis 12: e0007027.

27. World Health Organization, 2017. Trachoma Alternative Indicators Study Review: 31 August-1 September 2016. Geneva, Switzerland: WHO.

28. Fitzpatrick C, Asiedu K, Solomon AW, Mitja O, Marks M, Van der Stuyft $P$, Meheus F, 2018. Prioritizing surveillance activities for certification of yaws eradication based on a review and model of historical case reporting. PLoS Negl Trop Dis 12: e0006953.

29. Rostopira N, Tkacikova L, Rayevska G, Pylypenko V, Mikula I, Spivak M, 2003. Elaboration of enzyme immunoassay based on recombinant antigens and intended for diagnostics of syphilis. Folia Microbiol (Praha) 48: 549-553.

30. Solomon AW et al., 2015. The Global Trachoma Mapping Project: methodology of a 34-country population-based study. Ophthalmic Epidemiol 22: 214-225. 